\title{
Atrial and brain natriuretic peptides as markers of response to resynchronisation therapy
}

\author{
S G Molhoek, J J Bax, L van Erven, M Bootsma, P Steendijk, E Lentjes, E Boersma, \\ A van der Laarse, E E van der Wall, M J Schalii
}

Heart 2004:90:97-98

b ardiac resynchronisation therapy (CRT) has recently been introduced to treat patients with drug refractory heart failure. ${ }^{12}$ Studies have demonstrated immediate haemodynamic improvement after CRT, followed by improvement in symptoms, quality of life, and exercise capacity. ${ }^{12}$ Although the majority of patients respond well to CRT, in $20 \%$ of patients symptoms do not improve. The main problem is the lack of objective parameters to measure the effect of CRT.

Natriuretic peptides are now used in studies involving patients with heart failure. ${ }^{3}$ The value of these markers to objectively assess response to CRT was evaluated in this study.

\section{METHODS}

Based on traditional selection criteria (New York Heart Association (NYHA) functional class III-IV, left ventricular ejection fraction (LVEF) $<35 \%$, QRS duration $>120 \mathrm{~ms}$, and left bundle branch block configuration), 30 consecutive patients, of whom 23 were men (mean (SD) age 65 (12) years), underwent biventricular pacemaker implantation; 13 had ischaemic and 17 had idiopathic dilated cardiomyopathy. Medication consisted of diuretics, angiotensin converting enzyme inhibitors, spironolactone, $\beta$ blockers, and/or amiodarone, and remained unchanged during the entire study.

The day before implantation, echocardiography was performed in combination with tissue Doppler imaging (TDI) (to assess left ventricular dyssynchrony). Clinical evaluation included assessment of NYHA class, ECG (QRS duration, morphology), quality of life, and six minute walking distance. Blood samples were obtained for the analysis of atrial natriuretic peptide (ANP) and brain natriuretic peptide (BNP). The evaluation was repeated seven months after CRT.

Using a commercially available system (Vingmed system FiVe, General Electric-Vingmed, Milwaukee, Wisconsin, USA), standard two dimensional and colour Doppler data were saved in cineloop format. The LVEF was calculated using the biplane Simpson's rule. The severity of mitral regurgitation was graded semi-quantitatively from colour flow Doppler in the conventional parasternal long axis and apical four chamber images. Tissue Doppler parameters were measured from colour images by off line analysis. The digital cineloops were analysed using commercial software (Echopac 6.1, General Electric-Vingmed). The sample volume was placed in the basal portions of the septal and lateral walls (using the four chamber images), to derive velocity profiles. Timing of peak systolic velocity was measured from the onset of the QRS complex to the peak systolic velocity and expressed in milliseconds (ms). The difference between the timing of the peak systolic velocities of the septum versus the lateral wall, indicating dyssynchrony between the septum and lateral wall, was measured.
Peripheral venous blood was collected in sampling tubes containing EDTA as the anticoagulant. Plasma was separated and stored at $-80^{\circ} \mathrm{C}$ until the measurement of $\mathrm{N}$ terminal ANP and $\mathrm{N}$ terminal BNP concentrations by immunoassay ( $\mathrm{N}$ terminal-ANP ELISA (1-98) code number BI-20892 and $\mathrm{N}$ terminal-BNP ELISA (1-76), code number BI-20852; Biomedica, Vienna, Austria).

Data were expressed as mean (SD). Comparison of data was performed using the Student $t$ test for paired and unpaired data when appropriate. Since BNP and ANP are not normally distributed, we applied a log transformation. Paired Student's $t$ tests were applied to evaluate differences in the log transformed BNP and ANP concentrations before and after CRT. Analysis of variance was applied to evaluate BNP and ANP patterns between responders and non-responders.

Univariate analysis for categorical variables was performed using the $\chi^{2}$ test with Yates' correction. Simultaneous comparison of $>2$ mean values was performed by using one way analysis of variance (ANOVA) with Bonferoni correction. For all tests a probability value of $\mathrm{p}<0.05$ was considered significant.

\section{RESULTS}

The QRS duration was 176 (22) ms. The mean NYHA class was $3.1(0.3)$, the quality of life score was 39 (18), and the six minute walking distance was 259 (130) $\mathrm{m}$.

LVEF was 21 (9\%) (range 12-33\%). Five patients had 4+, five had $3+$, and seven had $2+$ mitral regurgitation. TDI demonstrated a delay between peak systolic velocity in the septum and the lateral wall of 72 (30) ms, illustrating LV dyssynchrony.

Plasma concentrations of ANP and BNP were 10522 (8091) pmol/l and 1242 (955) pmol/l, respectively.

Based on the improvement in NYHA class at seven months, patients were divided into responders $(\mathrm{n}=20)$ and nonresponders $(\mathrm{n}=10)$. Baseline characteristics were not different between the two groups (table 1). All echocardiographic results at baseline were comparable, except for the left ventricular dyssynchrony: responders exhibited a larger delay between the septum and lateral wall, as compared to non-responders (table 1). Mean plasma concentrations of peptides were not different between the two groups (table 1).

The improvement in symptoms was accompanied by an improvement in quality of life score (from 39 (15) to 28 (15), $\mathrm{p}<0.01$ ), six minute walking distance (from 264 (106) $\mathrm{m}$ to 385 (122) $\mathrm{m}, \mathrm{p}<0.01$ ), and LVEF (from $22(7 \%)$ to $31(9 \%$ ), $\mathrm{p}<0.05)$. Mitral regurgitation grade $3+$ or $4+$ was present in six patients and improved by $\geqslant 1$ grade in five. TDI showed

Abbreviations: ANP, atrial natriuretic peptide; BNP, brain natriuretic peptide; CRT, cardiac resynchronisation therapy; NYHA, New York Heart Association; LVEF, left ventricular ejection fraction; TDI, tissue Doppler imaging 
Table 1 Baseline characteristics of responders and nonresponders

\begin{tabular}{|c|c|c|c|}
\hline & $\begin{array}{l}\text { Responders } \\
(n=20)\end{array}$ & $\begin{array}{l}\text { Non-responders } \\
(n=10)\end{array}$ & p Value \\
\hline Male/female & $15 / 5$ & $8 / 2$ & NS \\
\hline Age (years) & $67(9)$ & $60(16)$ & NS \\
\hline \multicolumn{4}{|l|}{ Aetiology } \\
\hline non-ischaemic) & $8 / 12$ & $5 / 5$ & NS \\
\hline QRS (ms) & $178(21)$ & $172(25)$ & NS \\
\hline NYHA class & $3.15(0.4)$ & $3.0(0.0)$ & NS \\
\hline QoL score & $39(15)$ & $40(24)$ & NS \\
\hline $6 \mathrm{~min}$ WT $(\mathrm{m})$ & $26(106)$ & $258(192)$ & NS \\
\hline LVEF (\%) & $22(7)$ & $18(12)$ & NS \\
\hline MR grade $3-4+$ & $6(30 \%)$ & $4(40 \%)$ & NS \\
\hline S-L dyssync (ms) & $90(20)$ & $37(11)$ & $<0.01$ \\
\hline \multirow{3}{*}{$\begin{array}{l}\mathrm{N} \text { terminal ANP } \\
\text { (pmol/I) } \\
\mathrm{N} \text { terminal BNP } \\
\text { (pmol/l) }\end{array}$} & $11184(7339)$ & 9198 (9711) & NS \\
\hline & & & \\
\hline & $1368(761)$ & 989 (1269) & NS \\
\hline
\end{tabular}

ANP, atrial natriuretic peptide; BNP, brain natriuretic peptide; LVEF, left ventricular ejection fraction; MR, mitral regurgitation; NS, not significant; NYHA, New York Heart Association; QoL, quality of life; S-L dyssync, difference between septal and lateral peak systolic velocities, indicating dyssynchrony within the left ventricle; 6 min WT, 6 minute walk test.

an improvement of dyssynchrony and the delay in peak systolic velocities between the septum and lateral wall improved from $90 \quad(20) \mathrm{ms}$ to $44 \quad(28) \mathrm{ms} \quad(\mathrm{p}<0.01)$. Plasma concentrations of ANP and BNP decreased significantly (fig 1).

Fifteen of $20(75 \%)$ of responders showed more than a 10\% reduction in ANP or BNP plasma concentrations.

No improvement was seen in quality of life scores, exercise capacity, or LVEF; mitral regurgitation grade $3+$ or $4+$ was present in four patients and none improved at follow up. TDI did not show improvement in left ventricular dyssynchrony. In the non-responders, plasma concentrations of ANP and BNP did not improve (fig 1).

Nine of $10(90 \%)$ of non-responders did not show a reduction in ANP or BNP plasma concentrations of more than $10 \%$.

\section{DISCUSSION}

The majority of patients with end stage heart failure benefit from CRT, although objective assessment of response is difficult. $^{12}$ Studies have used improvement in NYHA class, which is a rather subjective parameter, as a marker of benefit. More objective parameters, such as quality of life score, six minute walking distance, and LVEF have also been used. ${ }^{2}$

In search of additional parameters to address severity of heart failure, natriuretic peptides have been used. ${ }^{3}$ In the current study patients with an improvement in clinical status showed a reduction in ANP and BNP plasma concentrations after CRT, suggesting the usefulness of natriuretic peptides as an objective and quantitative marker to evaluate response to CRT.

Natriuretic peptides may be even more useful as prognostic markers than as diagnostic markers. Omland and colleagues have shown that raised plasma concentrations of natriuretic peptides, in particular BNP, were predictive of mortality. ${ }^{4}$ The potential prognostic value of natriuretic peptides in patients treated with CRT therapy needs further study.

\section{Authors' affiliations}

S G Molhoek, J J Bax, L van Erven, M Bootsma, P Steendijk, E Lentjes, A van der Laarse, E E van der Wall, M J Schalii, Department of Cardiology, Leiden University Medical Center, The Netherlands
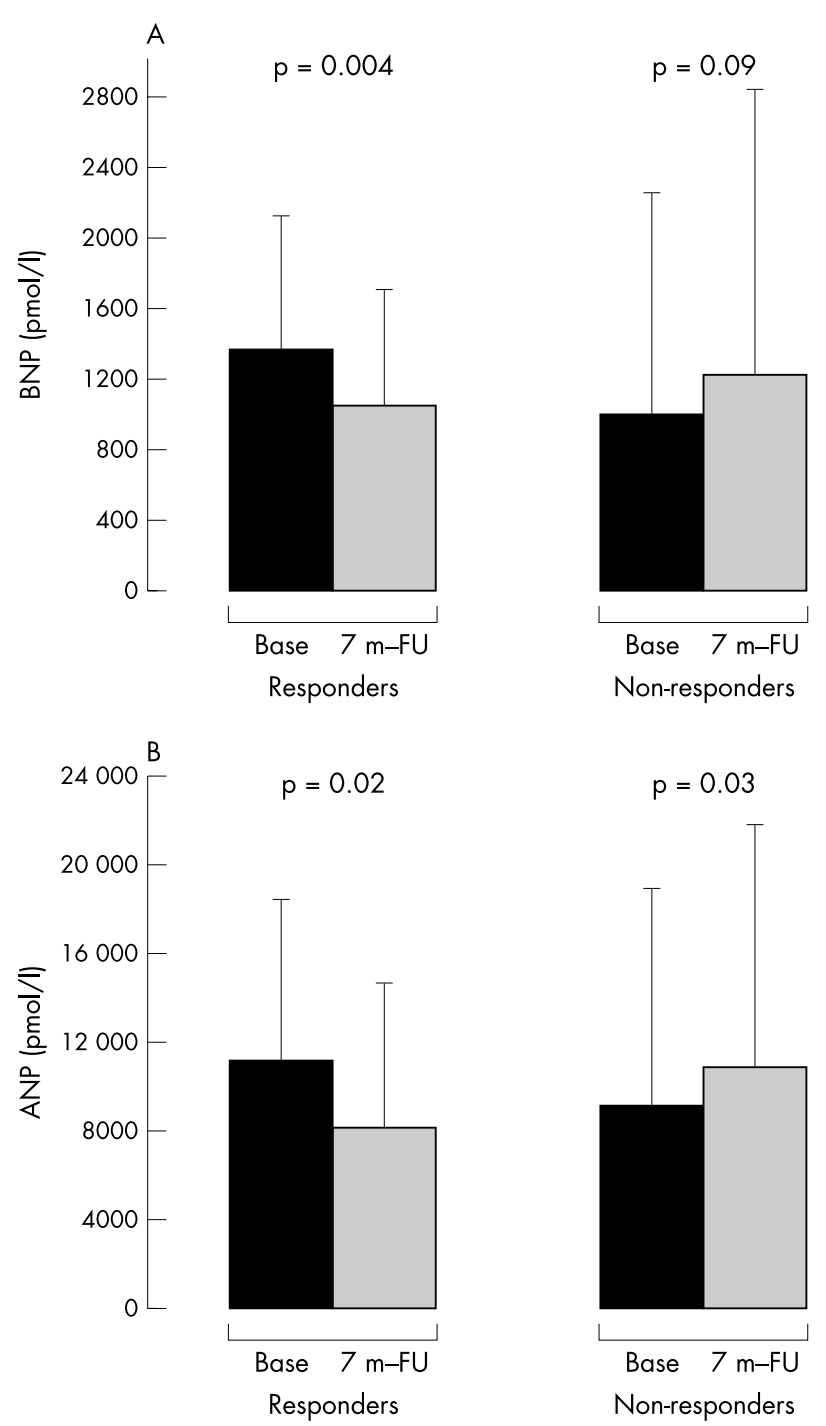

Figure 1 (A) Mean $N$ terminal BNP concentrations (A) and ANP concentrations $(B)$ in responders and non-responders before (Base) and after seven months $(7 \mathrm{~m}-\mathrm{FU})$ of cardiac resynchronisation therapy. ANP, atrial natriuretic peptide; BNP, brain natriuretic peptide.

E Boersma, Department of Epidemiology and Statistics, Erasmus University Rotterdam, The Netherlands

Correspondence to: Dr Sander G Molhoek, Albinusdreef 2 Leiden, 2300RC, The Netherlands; s.g.molhoek@lumc.nl

Accepted 4 July 2003

\section{REFERENCES}

1 Abraham T, Fisher AWG, Smith AL, et al. Cardiac resynchronization in chronic heart failure. N Engl J Med 2002;346:1845-53.

2 Cazeau S, Leclercq C, Lavergne T, et al. Effects of multisite biventricular pacing in patients with heart failure and intraventricular conduction delay. N Engl J Med 2001;344:873-80.

3 Levin ER, Gardner DG, Samson WK. Natriuretic peptides. N Engl J Med 1998;339:321-8.

4 Omland T, Aakvaag A, Bonarjee VV, et al. Plasma BNP as an indicator of left ventricular function and long term survival after acute myocardial infarction: Comparison with plasma atrial natriuretic peptide and $\mathrm{N}$ terminal proatrial natriuretic peptide. Circulation 1996;96:1963-9. 